\title{
Non-Cognitive Factors Affecting the Academic Performance of Fourth Year College Students of a Private College in Manila
}

\author{
By Maria Pamela Magpily* \\ Joseph Mercado ${ }^{+}$
}

The paper explored the relationships between general weighted average (GWA) and type of parents' employment and educational attainment, type of family structure, study habits, nutrition, and extracurricular activities of fourth year college students aged 19-20. Using a stratified random sample, the population was divided into subpopulations or strata. Findings showed that a higher GWA was associated with length of study before break, regular supper intake, frequency of exercise, weekend activity, and extracurricular activities. The findings suggest that frequent breaks during study time, regular supper intake, participating in extracurricular activities in school, boosting level of exercise, and arranging a weekend activity may sharpen college students' performance in school. The significant finding of a positive link between regular supper intake and academic performance in college students is something new and surprising given the importance that most literature place on eating breakfast to start the day. Apparently, the relationship between regular supper intake and academic performance in university students has received little attention from practitioners and researchers in the field of education.

Keywords: academic performance, regular supper intake, university students

\section{Introduction}

Academic performance remains to be measured through the ordinal scale of general weighted average (GWA) and continues to be the best predictive variable pertaining to academic success. A student is often labeled as high achieving, average, and low achieving based on his or her GWA.

This study is concerned with determining the non-cognitive factors that affect the academic performance of fourth year college students of College of the Holy Spirit Manila, as these would be significant in helping them achieve academic excellence and eventually fruitfully contributing members of the society. The students' academic achievement plays an important role in producing the best quality graduates who will become great leaders and

\footnotetext{
* Coordinator, College of the Holy Spirit Manila, Philippines.

${ }^{\dagger}$ Vice President for Branches and Campuses of Polytechnic University of the Philippines Philippines.
} 
manpower for the country contributing to the nation's economic and social development (Ali, Jusoff, Ali, Mokhtar, \& Salamt, 2009).

Dezmon (1995) proposed that academic achievement is associated with cognitive as well as non-cognitive variables. The cognitive variables are the academic grades, class ranks, test scores, and scholastic aptitude test scores. There are however, other non-cognitive variables associated with the academic achievements of students that are of much significance.

The word non-cognitive has been employed in the fields of economics and sociology more extensively than in the domains of psychology and education, and it is used quite broadly, "as a catch-all ... to focus on variables other than those measured by test scores" (Farkas, 2003, p. 542). It is believed that noncognitive rather than cognitive factors are the more conspicuous elements of success at both school and work (Bowles \& Gintis, 2002; Farkas, 2003).

"Most of the effects of schooling on occupational and earnings attainment are due, not to the effects of schooling on cognitive skills as measured by test scores, but to the correlation between schooling and various non-cognitive traits" (Farkas, 2003, p. 547).

Arlan, Shrestha, and Wingo (2008) determined the causes that affect the academic performance of Overseas Filipino Workers' children in Lyceum of the Philippines University. Findings revealed that migration of parents did not affect the academic performance of children.

Chiu et al. (2012) demonstrated that the education level of the students' fathers had the greater impact on academic achievement. This contradicted the results of the study by Ortiz and Dehon (2008) that the fathers' occupational activity and the mothers' level of education (Ortiz \& Dehon, 2008; Hijazi \& Naqvi, 2006) seemed to be most influential. Karemera, Reuben and Sillah Sillah (2003) found that the level of education and social status of parents were factors conducive to positive college experience and learning.

$\mathrm{Wu}$, Schimmelle, Hou, and Ouellet (2012) verified that youth from intact families had a consistent advantage over those from fragile families, including cohabiting-parent households.

Costa (2009) found that sleep had no strong relationship to grade point average (GPA). Her findings ran contrary to the data of Pilcher and Walters (1997), who found sleep deprivation to negatively affect performance and to the findings of Kelly, Kelly, and Clanton (2001), who found length of sleep among undergraduate students to be related to GPA.

Nonis and Hudson (2006) stated that the amount of time spent studying or at work had no direct influence on academic performance. Hijazi and Naqvi (2009) gathered similar data that spending more study hours is not significant as far as student performance is concerned. However, results of both studies contradict the findings of the survey done by Dobson (2011) that students who spent more than 5 hours per week doing schoolwork had a higher GPA than those who spent 5 hours or less per week.

Wang and Shiveley (2009) and Dobson (2011) found that students who are involved in extracurricular activities and regularly participated in vigorous 
physical activity had higher GPAs (Pate, Heath, Dowada, \& Trost, 1996; Parker-Pope, 2010) than those who are not involved in such activities.

According to Science Daily (2013), every organ has a clock that means there are times that our livers, intestines, muscles, and other organs will work at peak efficiency and other times when they are - more or less - sleeping. While many people refer to circadian rhythms as a single process, there are actually a number of body clocks that oscillate throughout the day. For example, mental alertness tends to peak twice in a day at 9 am and $9 \mathrm{pm}$, while physical strength tends to crest at 11 am and 7 pm (Cherry, 2013).

The brain is one master controller of circadian rhythm. The cues of light and energy intake are processed in the brain through the eyes and in the liver, the bodies' main metabolic sensor. According to Maret (2013), the proper functioning of the eyes, the heart, the brain, the gonads, the joints, and the kidneys are dependent on good liver activity.

Maret (2013) added that the liver is most active in rebuilding the body during the night. The Natural Recovery Plan, in 2013, mentioned that the cycle could be regarded as running from 3 am when liver time concludes and the body's energies turn outward in readying the body for the day by cleansing the lungs and the large intestine. Then, from $3 \mathrm{pm}$ onward, the energies flow back in to restore and maintain the body during rest and sleep.

According to Stokkan, Yamazaki, Tei, Sakaki, and Menaker (2001), the liver is susceptible to the feeding pattern of mammals when establishing its local clock system and therefore the metabolism of a mammal is completely dependent on its environment. Those metabolic cycles are critical for processes from cholesterol breakdown to glucose production, and they should be primed to turn on when we eat and back off when we do not or vice versa (Science Daily, 2013).

Adolphus, Lawton, and Dye (2013) stated that children may be particularly vulnerable to the nutritional effects of breakfast on brain activity and associated cognitive, behavioral, and academic outcomes because they have a higher brain glucose metabolism compared to adults. Positron Emission Tomography studies show that cerebral metabolic rate of glucose utilization is approximately twice as high in children aged 4-10 years compared to adults. This higher rate of glucose utilization gradually declines from age 10 and usually reaches adult levels by the age of 16-18 years (Chugani, 1998). Average cerebral blood flow and cerebral oxygen utilization is 1.8 and 1.3 times higher in children aged 3-11 years compared to adults, respectively (Kennedy \& Sokoloff, 1957; Chiron et al., 1992). Moreover, the longer overnight fasting period due to higher sleep demands during childhood and adolescence compared to adults can deplete glycogen stores overnight (Thorleifsdottir, Björnsson, Benediktsdottir, Gislason, \& Kristbjarnarson, 2002). To maintain this higher metabolic rate, a continuous supply of energy derived from glucose is needed; hence breakfast consumption may be vital in providing adequate energy in the morning.

The purpose of the study by Kim et al. (2003) was to obtain a fuller understanding of the association of dietary behaviors, physical status, and 
socio-economic status with academic performance in Korean teenagers. The subjects in the study were 6,463 boys and girls in grades 5 (10-11 years old), 8 (13-14 years old), and 11 (16-17 years old). The academic performance of students was strongly associated with dietary behaviors, especially with regularity of three meals even after control for parents' education level. Regular breakfast and lunch were more important in grades 5 and 8, while regular dinner was more related with academic performance in grade 11 . These results were the first to demonstrate an association between regularity of all three meals and academic performance. This study showed that not only regular breakfast, but also regular lunch and dinner are associated with academic functioning of teenagers. However, the reason why regular meals are related to academic performance was not clear.

In 2002, Hanson and Austin's analytic sample consisting of 1,395 schools found that students in grades 7, 9, and 11 who ate breakfast on the day of the survey had higher Academic Performance Index (API) scores. In 2011, Dobson's survey showed that undergraduate students from the Business and Arts and Social Sciences at a public research university in Canada who ate their breakfast regularly had higher GPAs than those who did not.

\section{Methods}

This is a descriptive study that investigated the non-cognitive factors affecting the academic performance of fourth year college students.

A stratified random sample was utilized. The population was divided into subpopulations or strata (i.e., low-achieving, average, and high-achieving students). A sample was drawn from each. Fifty-eight (58) students (10 low achievers, 42 average, and 6 high achievers) participated in the study.

A researcher-developed questionnaire consisting of 25 items relating to (a) type of parents' employment, (b) type of family structure, (c) study habits, (d) nutrition, and (e) extracurricular activities was utilized.

The researcher employed SPSS 17.0 to calculate the frequency, percentage, and rank of respondents' answers to each item. Pearson $r$ was utilized to test the significant relationship between two variables.

\section{Results}

The relationship between fathers' occupation and GWA is not significant. This disagrees with the findings of Ortiz and Dehon (2008) that the fathers' occupational activity affects academic performance, on the other hand, the result agrees with the findings of Arlan, Shrestha, and Wingo (2008) that migration of parent/s does not affect the academic performance of their children.

The relationship between highest education completed by father and GWA is not significant. This contradicts the findings of Chiu et al. (2012) that the 
fathers' education level had the greater impact on academic achievement and the study of Karemera et al. (2003) that parents' level of education affects academic performance.

The relationship between highest education completed by mother and GWA is not significant. This contradicts the findings of Hijazi and Naqvi (2006) that students who are performing well have educated mothers and the study of Ortiz and Dehon (2008) that the mothers' level of education affects academic performance.

The relationship between parents' marital status and GWA is not significant. This disagrees with the findings of Wu et al. (2012) that youth from intact families have a consistent advantage over those from fragile families, including cohabiting-parent households.

The relationship between number of hours of sleep and GWA is not significant. This agrees with the findings of Costa (2009) that did not show a significant relationship between sleep habits and GPA. The findings of the current study contradicts the data of Pilcher and Walters (1997), who found sleep deprivation to negatively affect performance and the findings of Kelly et al. (2001), who found length of sleep among undergraduate students to be related to GPA.

The relationship between hours per week of study outside class and GWA is not significant. This agrees with the results of the study of Nonis and Hudson (2006) and Hijazi and Naqvi (2006) that the amount of time spent studying had no direct influence on academic performance. These findings however, contradict the result of the survey done by Dobson (2011) that students who spend more than 5 hours per week doing schoolwork have a higher GPA than those who spend 5 hours or less per week.

The relationship between length of study before break and GWA is significant. This result agrees with the findings of Waterworth (2003) that studying continuously for an average of 8-9 hours per day may create fatigue and overall exertion among students, which may lead to lower performance on examinations.

The relationship between regular breakfast intake and GWA is not significant. This contradicts the survey of Dobson (2011) that showed undergraduate students who eat their breakfast regularly tend to have higher GPAs than those who do not eat their breakfast regularly; the findings of Boschloo et al. (2012) that breakfast skipping (eating breakfast less than 5 days a week) was associated with lower average annual school grades in a sample of 605 Dutch adolescents aged 11-18 years old; the survey of Hanson and Austin (2002) that indicated schools with higher percentages of students in grades 7, 9, and 11 who ate breakfast on the day of the survey had higher Academic Performance Index scores; and 21 other studies that demonstrated habitual breakfast (frequency and quality) and school breakfast programs had a positive effect on children and adolescents' academic performance (Adolphus et al., 2013).

Breakfast is regarded by many as "the most important meal of the day" because an adequate food intake at the beginning of the day helps to ensure that 
nutrient needs by the body for the remainder of the day are more likely to be met (Scragg, Quigley \& Taylor, 2007). However, Adolphus et al. (2013) stated that children may be particularly vulnerable to the nutritional effects of breakfast on brain activity and associated cognitive, behavioral, and academic outcomes. Children have higher brain glucose metabolism compared with adults. Cerebral metabolic rate of glucose utilization is approximately twice as high in children aged 4-10 years compared with adults. This higher rate of glucose utilization gradually declines from age 10 and usually reaches adult levels by the age of 16-18 (Chugani, 1998). Average cerebral blood flow and cerebral oxygen utilization is 1.8 and 1.3 times higher in children aged 3-11 years compared with adults, respectively (Kennedy \& Sokoloff, 1957; Chiron et al., 1992). The longer overnight fasting period due to higher sleep demands during childhood and adolescence compared with adults can deplete glycogen stores overnight (Thorleifsdottir et al., 2002). To maintain this higher metabolic rate, a continuous supply of energy derived from glucose is needed; hence breakfast consumption may be vital in providing adequate energy in the morning.

It is important to note that the respondents in the present study were fourth year college students who were aged 19-20. The age factor could be the reason why a regular breakfast intake did not have a significant relationship with GWA. The respondents in the studies of Dobson (2011), Boschloo et al. (2012), Hanson and Austin (2002), and articles reviewed by Adolphus et al. (2013) were younger.

The relationship between regular lunch intake and GWA is not significant. This finding agrees with the result of the study of Kim et al. (2003) that regular breakfast and lunch were more important in grades 5 and 8 than grade 11 students.

The relationship between regular supper intake and GWA is significant. Kim et al. (2003) found a weak association of having a regular dinner in grades 5 and 8 boys, but having a regular dinner had the strongest association with academic performance among the three meals in grade 11 .

The relationship between extracurricular activities in school and GWA is significant. This result agrees with the findings of Wang and Shiveley (2009) that students achieved much higher rates of graduation, maintained better GWAs, and had higher good standing rates when they engaged in extracurricular activities. Participation in extracurricular activities did not lower the academic performance of participants, but instead helped them to persist in college and bolstered their progress toward graduation. Dobson (2011) also stated that students who are involved in extracurricular activities have higher GPAs than those who are not involved in such activities.

The relationship between after school job and GWA is not significant. This result does not support the findings of Klott (1998) that working long hours at a job during the school year often has a detrimental effect on academic performance. The empirical evidence in the study of Andreopoulos, Antoniou, Panayides, and Vassiliou (2008) did not support the assumption that a full time working student will show a lower academic performance 
relative to a part time working student or a full time student because other variables affect academic performance such as talent, motivation, ambition, and efficiency of study time.

Table 1. Correlation between Non-Cognitive Factors and GWA

\begin{tabular}{|c|c|c|c|}
\hline & $\begin{array}{l}\text { Computed } \\
\text { Pearson } r\end{array}$ & P-value & Decision \\
\hline \multicolumn{4}{|c|}{ A. Parents' Type of Employment and Education and GWA } \\
\hline Fathers' Occupation & -0.195 & 0.142 & Accept HO \\
\hline Mothers' Occupation & 0.103 & 0.441 & Accept HO \\
\hline Fathers' Highest Education & -0.033 & 0.804 & Accept HO \\
\hline Mothers' Highest Education & 0.039 & 0.773 & Accept HO \\
\hline \multicolumn{4}{|l|}{ B. Type of Family Structure and GWA } \\
\hline Students' Residence & 0.000 & 1.000 & Accept HO \\
\hline Parents' Marital Status & -0.072 & 0.591 & Accept HO \\
\hline Type of Family Structure & -0.081 & 0.547 & Accept HO \\
\hline Birth Order & 0.090 & 0.500 & Accept HO \\
\hline \multicolumn{4}{|l|}{ C. Study Habits and GWA } \\
\hline Number of Hours of Sleep & 0.045 & 0.738 & Accept HO \\
\hline Hours per Week of Study Outside Class & 0.188 & 0.158 & Accept HO \\
\hline Specific Place of Study & 0.249 & 0.059 & Accept HO \\
\hline Regular Time of Study & -0.003 & 0.984 & Accept HO \\
\hline Method of Study & -0.115 & 0.389 & Accept HO \\
\hline Length of Study Before Break & $0.383^{* *}$ & 0.003 & Reject HO \\
\hline \multicolumn{4}{|l|}{ D. Nutrition and GWA } \\
\hline Body Mass Index & 0.083 & 0.538 & Accept HO \\
\hline Regular Breakfast Intake & -0.186 & 0.162 & Accept HO \\
\hline Regular Lunch Intake & -0.180 & 0.175 & Accept HO \\
\hline Regular Supper Intake & $-0.499^{* *}$ & 0.000 & Reject HO \\
\hline Frequency of Snack Time & -0.107 & 0.422 & Accept HO \\
\hline On Diet & 0.080 & 0.549 & Accept HO \\
\hline \multicolumn{4}{|l|}{ E. Extracurricular Activities and GWA } \\
\hline Extracurricular Activities & $0.280^{*}$ & 0.033 & Reject HO \\
\hline Purpose of Extracurricular Activities & $0.267^{*}$ & 0.043 & Reject HO \\
\hline After School Job & -0.053 & 0.695 & Accept HO \\
\hline Frequency of Exercise & $0.394^{* *}$ & 0.002 & Reject HO \\
\hline Weekend Activity & $0.337^{* * 4}$ & 0.010 & Reject HO \\
\hline Frequency of Social Activity & -0.141 & 0.293 & Accept HO \\
\hline
\end{tabular}

The relationship between frequency of exercise and GWA is significant. This agrees with the findings of Pate et al. (1996) that exercise has an effect on academic performance and the results of the study of Parker-Pope (2010) that students who regularly participated in vigorous physical activity had higher GPAs. According to Valmon (2011), the brain is sharpened with physical exercise in the same way that muscles are strengthened.

The relationship between weekend activity and GWA is significant. 
According to the results of the study conducted by Yousuf, Sarwar, and Ranjha (2011), similar future plans of friends serve as inspirations for showing good performance at higher education and communication with peer groups as a helping matter clearly indicates that such interaction is helpful for promoting quality performance at higher education.

The relationship between mothers' occupation, students' residence, type of family, birth order, specific place, regular time, and method of study, body mass index, frequency of snack time, being on diet, and frequency of social activity and GWA is not significant (Table 1).

\section{Conclusions and Recommendations}

\section{Conclusions}

The parents' highest education, employment, and marital status, students' residence, type of family, birth order, number of hours of sleep, hours per week of study outside class, specific place, regular time, and method of study, body mass index, regular breakfast and lunch intake, frequency of snack time, diet after school job, and frequency of social activity do not affect college students' academic performance.

Regular supper intake affects college students' academic performance. Adults have a lower brain glucose metabolism compared to children. The rate of glucose utilization usually reaches adult levels by the age of 16-18 years (Chugani, 1998). Moreover, the proper functioning of the brain is dependent on good liver activity. Dupuis (2010) stated that the liver is most active in rebuilding the body from 1-3 am. According to Stokkan et al. (2001), the liver is susceptible to the feeding pattern of mammals when establishing its local clock system and therefore the metabolism of a mammal is completely dependent on its environment. Those metabolic cycles are critical for processes from cholesterol breakdown to glucose production, and they should be primed to turn on when we eat and back off when we do not, or vice versa.

Length of study before break affects academic performance. A break time while studying is necessary for refreshing individuals' mind and helping them enhance their overall performance. Students who regularly engage in vigorous exercise, participate in extracurricular activities, and enjoy weekend activities get better grades. Exercise helps establish body-mind balance. Extracurricular and weekend activities allow students to unwind. The results of the study support the belief that the health of the body is directly related to the cognitive abilities of the mind.

\section{Recommendations}

Knowledge that eating supper is important in achieving educational outcomes should be widely disseminated to parents, students, and schools. Teach students that eating dinner may sharpen their performance in school. Students should join extracurricular activities in school, arrange weekend 
activities, and boost their level of exercise to improve their grades. A good time for exercise is from 5-7 pm, when the kidney system, the root of our overall energy is most active (Dupuis, 2010). From 7-9 pm is a good time to start relaxing and unwinding from the day since the pericardium system is most active (Dupuis, 2010). An ideal time for students to study for an exam or to review their lessons is at $9 \mathrm{pm}$ when mental alertness tends to peak (Cherry, 2013). Even if the results of the study did not find a significant relationship between regular breakfast intake and academic performance, it is recommended that students eat breakfast particularly from 7-9 am when the stomach is most active (Dupuis, 2010) in order to sustain an adequate level of glucose and to minimize fluctuations between meals (Bellisle, 2004). Although the findings of the study did not prove causal relationships, it strongly suggests that academic functioning of university students is (at least in part) associated with regular supper intake. Research about this subject is still limited, but results seem promising, thus further studies are needed to find out more about the relationship between the two entities. Future research should have a larger sample size to reveal any small relationships that were not discovered throughout the course of this research. Under these circumstances, some of the relationships that were not significant in this study may be significant. Developing an experimental study to determine causal relationships would be ideal.

\section{Acknowledgement}

Our thanks to Republic of the Philippines, Office of the President, Commission on Higher Education Support for Paper Presentations in International Conferences.

\section{References}

Adolphus, K., Lawton, C., \& Dye, L. (2013). The effects of breakfast on behavior and academic performance in children and adolescents. Frontiers in Human Neuroscience, 7, 425.

Ali, N., Jusoff, K., Ali, S., Mokhtar, N., \& Salamt, A. (2009). The factors influencing students' performance at Universiti Teknologi MARA Kedah, Malaysia. Canadian Research \& Development Center of Sciences and Cultures, 3(4), 81-90.

Andreopoulos, G., Antoniou, E., Panayides, A., \& Vassiliou, E. (2008). The impact of employment and physical activity on academic performance. College Teaching Methods \& Styles Journal, 4(10). Retrieved from http://bit.ly/1fCJIch.

Arlan, K. Shrestha, J., \& Wingo, Y. (2008). Employment of Overseas Filipino Workers (OFWs) and its implications on the academic performance of their children. Retrieved from http://bit.ly/1OyedM6.

Bellisle, F. (2004). Effects of diet on behavior and cognition in children. British Journal of Nutrition, 2, S227-S232. 
Boschloo, A., Ouwehand, C., Dekker, S., Lee, N., De Groot, R., \& Krabbendam, L. (2012). The relation between breakfast skipping and school performance in adolescents. Mind Brain Education, 6, 81-88.

Bowles, S. \& Gintis, H. (2002). The inheritance of inequality. The Journal of Economic Perspectives, 16, 3-30.

Cherry, K. (2013). Circadian rhythms: The body's "clock". Retrieved from http://abt.cm/1giyKJQ.

Chiron C., Raynaud C., Maziere B., Zilbovicius M., Laflamme L., \& Masure M. (1992). Changes in regional cerebral blood flow during brain maturation in children and adolescents. Journal of Nuclear Medicine, 33, 696-703.

Chiu, J., Markson, C., Raicovi, V., Economos, J., Howell, C., Morote, E., \& Inserra, A. (2012). Which matters most? Perceptions of family income or parental education on academic achievement. Paper presented during the 2012 Hawaii International Conference on Education and International Business and Economy Conference.

Chugani, H. (1998). A critical period of brain development: Studies of cerebral glucose utilization with Positron Emission Tomography. Preventive Medicine, 27, 184-188.

Costa, A. (2009). The relationship between health and college students' grade point average. Retrieved from http://bit.ly/1CYP5NJ.

Dezmon, B. (1995). A comparative analysis of the relationship between student perceptions of school climate and academic achievement among high achieving versus low achieving African-American students in suburban middle schools (Doctoral dissertation). University of Maryland, Baltimore.

Dobson, D. (2011). Understanding the drivers of academic success in university students. Retrieved from http://bit.ly/1DLa95i.

Dupuis, C. (2010, April 4). Circadian rhythms, the Chinese clock and how to live in sync. Retrieved from http://bit.ly/1MM4tNz.

Farkas, G. (2003). Cognitive skills and noncognitive traits and behaviors in stratification processes. Annual Review of Sociology, 29, 541-562.

Hanson, T. \& Austin, G. (2002). Health risks, resilience, and the Academic Performance Index. (California Healthy Kids Survey Fact Sheet 1). Los Alamitos, CA: WestEd.

Hijazi, S. \& Naqvi, S. (2006). Factors affecting student's performance: A case of private colleges. Bangladesh e-Journal of Sociology, 3(1). Retrieved from http://bit.ly/1HXGzxq.

Karemera, D., Reuben, L., \& Sillah, M. (2003). The effects of academic environment and background characteristics on student satisfaction and performance: The case of South Carolina State University School of Business. College Student Journal, 37, 298-308.

Kelly, W., Kelly, K., \& Clanton, R. (2001). The relationship between sleep length and grade-point average among college students. College Student Journal, 35, 84-87.

Kennedy, C. \& Sokoloff, L. (1957). An adaptation of the nitrous oxide method to the study of the cerebral circulation in children - normal values for cerebral blood flow and cerebral metabolic rate in childhood. Journal of Clinical Investigation, 36, 1130-1137.

Klott, G. (1998). Teens' after-school jobs take huge academic toll. Retrieved from http://bit.ly/1HNsqmp.

Kim, H., Frongillo, E., Han, S., Oh, S., Kim, W., Jang, Y., \& Kim, S. (2003). Academic performance of Korean children is associated with dietary behaviors and physical status. Asia Pacific Journal of Clinical Nutrition, 12, 186-192. 
Maret, K. (2013). Importance of the liver for healing. Retrieved from http://bit.ly/1gRXgl9.

Nonis, S. \& Hudson, G. (2006). Academic performance of college students: Influence of time spent studying and working. Journal of Education for Business, 81(3), 151-159.

Ortiz, E. \& Dehon, C. (2008). What are the factors of success at university? A case study in Belgium. CESifo Economic Studies, 54(2), 121-148.

Parker-Pope, T. (2010, June 3). Vigorous exercise linked with better grades. The New York Times. Retrieved from http://nyti.ms/IL5QBs.

Pate, R., Heath, G., Dowada, M., \& Trost, S. (1996). Associations between physical activity and other health behaviors in a representative sample of US adolescents. American Journal of Public Health, 86(11), 1577-1581.

Pilcher, J. \& Walters, A. (1997). How sleep deprivation affects psychological variables related to college students' cognitive performance. Journal of American College Health, 46, 121-127.

Science Daily (2013). When you eat matters, not just what you eat. Retrieved from http://bit.ly/1BryWdB.

Scragg, R., Quigley, R., \& Taylor, R. (2007, May 23). Is consuming breakfast important for academic performance, maintaining a healthy body weight, and improving nutrient intake and lifestyle habits in children?. A report prepared by the Scientific Committee of the Agencies for Nutrition Action. Retrieved from http://bit.ly/1SbhBmc.

Stokkan, K., Yamazaki, S., Tei, H., Sakaki, Y., \& Menaker, M. (2001). Entrainment of the circadian clock in the liver by feeding. Science, 291(5503), 490-493.

Thorleifsdottir, B., Björnsson, J., Benediktsdottir, B., Gislason, T., \& Kristbjarnarson, H. (2002). Sleep and sleep habits from childhood to young adulthood over a 10year period. Journal of Psychosomatic Research, 53, 529-537.

Valmon, J. (2011). Factors that can affect a student's academic performance. Retrieved from http://bit.ly/1JiBUZi.

Wang, J. \& Shiveley, J. (2009). The impact of extracurricular activity on student academic performance. Retrieved from http://bit.ly/1IoByLl.

Waterworth, S. (2003). Time management strategies in nursing practice. Journal of Advanced Nursing, 43(5), 432-440.

$\mathrm{Wu}, \mathrm{Z}$., Schimmele, C., Hou, F., \& Ouellet, N. (2012). Family structure and university enrollment and completion. Retrieved http://bit.ly/1U3BM32.

Yousuf, M., Sarwar, M., \& Ranjha, A. (2011). A study of non-cognitive variables of academic achievement at higher education: Nominal group study. Asian Social Science, 7(7), 53-58. 
